Bacher Ulrike (Orcid ID: 0000-0001-8771-947X)

Pabst Thomas (Orcid ID: 0000-0002-6055-5257)

\title{
Autologous stem cell transfusions on multiple days in patients with multiple myeloma - does it matter?
}

\author{
Letter to the Editor
}

Authors: ${ }^{1}$ Sebastian Moser ${ }^{*}{ }^{2}$ Ulrike Bacher*, ${ }^{1}$ Barbara Jeker, ${ }^{2}$ Behrouz Mansouri Taleghani, ${ }^{3}$ Daniel Betticher, ${ }^{4}$ Axel Ruefer, ${ }^{5}$ Thomas Egger, ${ }^{1}$ Urban Novak, ${ }^{1}$ Thomas Pabst.

*These authors contributed equally and should both be considered first authors.

Affiliations: ${ }^{1}$ Department of Medical Oncology; and ${ }^{2}$ Department of Hematology and Central Hematology Laboratory; Inselspital, University Hospital and University of Bern; Bern; Switzerland; ${ }^{3}$ Department of Oncology; Kantonsspital, Fribourg; ${ }^{4}$ Department of Hematology; Kantonsspital, Lucerne; and ${ }^{5}$ Department of Oncology; Bürgerspital, Solothurn; Switzerland.

Manuscript Information: total word count: 999 words; 0 table, 1 figure, 2 supplemental figures; 3 supplemental tables; 10 references.

Running title: Autologous transplantation on multiple days in myeloma.

Key Words: myeloma, autologous stem cell transplantation, poor mobilization, multiple day transplantation, CD34+, prognosis, outcome.

Conflict of interest: All authors declare no conflict of interest.

Funding: A grant of the Swiss Cancer League (SCL \#38-167 to TP) supported this work.

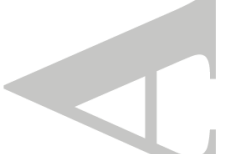

This article has been accepted for publication and undergone full peer review but has not been through the copyediting, typesetting, pagination and proofreading process which may lead to differences between this version and the Version of Record. Please cite this article as doi: 10.1002/hon.2673 
Corresponding author: Thomas Pabst, MD; Extraordinary Professor; Department of Medical Oncology; University Hospital and University of Bern; and Center for Hemato-Oncology; University Cancer Center; 3010 Berne; Switzerland.

Autologous stem cell transplantation (ASCT) following high-dose chemotherapy (HDCT) is a cornerstone of first-line treatment in myeloma patients. ${ }^{1}$ Freezing of the hematopoietic stem cells (HSC) to bypass the time between stem cell collection and completion of HDCT is crucial. Due to the vulnerability of HSC to the freezing process, anti-freezing agents are mandatory, and between 5 and 10 vol\% dimethyl-sulfoxide (DMSO) is commonly added. ${ }^{2-4}$ However, DMSO exerts toxic effects including nausea, vomiting, sedation and headaches, ${ }^{4-5}$ and triggers histamine release resulting in hypo-/hypertension, cardiac arrhythmia, rashes, renal failure, pulmonary edema, and bronchospasms. As toxicity of DMSO is dose-related, the recommended dose is $1 \mathrm{~g} / \mathrm{kg}$ body weight (b.w.). ${ }^{4}$

To provide reliable engraftment, a minimum of $2.0 \times 10^{6} \mathrm{CD} 34+\mathrm{HSC} / \mathrm{kg}$ b.w. is required. Usually, this number is collected during a single apheresis procedure, whereas some patients fail to reach this goal with a single-day apheresis procedure. Advanced age, high bone marrow infiltration, and a history of multiple induction lines including stem cell damaging compounds are associated with poor HSC mobilization. ${ }^{6}$ In patients with poor CD34+ mobilization at the first apheresis day, additional apheresis procedures are needed. This implies additional DMSO doses eventually exceeding the recommended maximum daily dose at retransfusion. In such patients, washing the autograft before transplantation or a reduced infusion rate can reduce side effects caused by increased DMSO administration. ${ }^{7-9}$

At our center, the maximum transplantation volume is $300 \mathrm{~mL}$ per day with a DMSO concentration of $5 \%$ while avoiding washing and concentration steps. For patients with transfusion volumes exceeding these limits, ASCT is split over several days. At present, it remains unclear whether splitting the transplantation procedure is similarly beneficial as compared to a single day procedure, and the possible impact on outcome and engraftment is hardly studied. We retrospectively evaluated the effect of ASCT split over multiple days on survival outcomes and hematological recovery compared to a single day procedure in a large cohort of myeloma patients.

Our study analyzed all consecutive myeloma patients undergoing first ASCT at the University Hospital Bern between 01/2006-12/2014 (Table S1). Patients received non-myelosuppressive mobilization chemotherapy at day +1 with vinorelbine or, alternatively, gemcitabine in patients with concurrent peripheral neuropathy (Table S2). Starting on day +4 , patients received GCSF s.c. In patients with peripheral CD34+ counts $<10 / \mu \mathrm{L}$ at day +8 , the CXCR 4 antagonist 
plerixafor was added at day +9 . HDCT was standard $200 \mathrm{mg} / \mathrm{m}^{2}$ melphalan followed by ASCT. Patients were offered maintenance therapy with $10 \mathrm{mg}$ lenalidomide continuously for two years post-transplant. All patients gave written informed consent, and the ethics committee of Bern, Switzerland approved the study (KEK BE \# 374/15).

Between 2006 and 2014, 271 patients with multiple myeloma (265 patients), plasma cell leukemia (4 patients), or POEMS syndrome (2 patients) underwent a first ASCT. The majority (244 patients; 90\%) received ASCT within a single day, and this group was termed $\mathrm{Tx}_{1}$. The $\mathrm{Tx}_{2-3}$ group comprised 23 patients receiving HSC on two days and four patients on three days. Both groups $\left(\mathrm{Tx}_{1}\right.$ and $\left.\mathrm{Tx}_{2-3}\right)$ did not differ in clinical characteristics at diagnosis.

Characteristics regarding HSC mobilization and CD34+ collection are summarized in Table S2. Plerixafor tended to be administered more frequently in $\mathrm{Tx}_{2-3}$ than $T x_{1}(p=0.0715)$. At the day of HSC collection, peripheral CD34+ counts were lower in $\mathrm{Tx}_{2-3}$. As expected, the final transplanted autograft volume was higher in $\mathrm{Tx}_{2-3}$ patients. The total amount of transplanted CD34+ cells/kg b.w. was lower in the $\mathrm{Tx}_{2-3}$ group, suggesting higher dilution of CD34+ cells $(p<0.0001)$. The median recovery (Table S3) for neutrophils was 18 days for $\mathrm{Tx}_{2-3}$ and 12 days for $\operatorname{Tx}_{1}(p=0.0048)$, and for platelets 18 versus 14 days $(p=0.0004)$. Tx $x_{2-3}$ patients had longer median hospitalization duration (23 versus 19 days; $p=0.0006$ ), and fever was more frequent in $\operatorname{Tx}_{2-3}(96 \%$ versus $76 \% ; p=0.0131)$.

The median follow-up for all patients was 56 months. Relapse-free survival (RFS) was 39 months, and 169 relapses (62\%) occurred so far. Median overall survival (OS) was 91 months, and 82 patients (30\%) have died during follow-up. Tx $x_{2-3}$ patients had shorter median RFS (21 versus 40 months for $\mathrm{Tx}_{1} ; \mathrm{p}=0.0245$ ), and shorter median OS with (55 versus 93 months; $\mathrm{p}=0.0134$ ) (Table S3, Figure 1). The impact of the ISS staging system on survival outcomes is given in Figure $\mathbf{S 1}$.

Maintenance with thalidomide or lenalidomide was administered at similar frequencies in $\mathrm{Tx}_{2}$ ${ }_{3}$ and $\mathrm{Tx}_{1}(56 \%$ and $44 \% ; \mathrm{p}=0.3106)$, and a second ASCT was similarly performed $(26 \%$ and $35 \% ; p=0.3795)$. Survival outcomes were not affected whether patients had a subsequent second ASCT or not (Figure S2).

Obviously, there are alternatives to a multiple day transplantation procedure in patients with poor CD34+ mobilization. Among them, the autograft can be washed and concentrated to reduce the volume. Inevitably, this is associated with loss of CD34+ cells. Also, many centers perform single day procedures independently of the volume of the autograft. As demonstrated in a recent EBMT survey, the majority of transplant centers continue to use $10 \%$ DSMO for ASCT in myeloma and lymphoma patients, but a proportion of centers either use lower concentrations, $5 \%$ or $7.5 \%$, or they wash and concentrate the cells before infusion. ${ }^{4}$ 
Furthermore, this survey indicated that patients in the highest quartile receiving DSMO had significantly more side effects. Better standardization of the preservation strategy with reduced DSMO concentration and increased awareness of DSMO dose-dependent toxicity was suggested to reduce toxicity and morbidity of the transplant procedure.

In conclusion, multiple day transplantation was observed in roughly $10 \%$ of myeloma patients undergoing HSCT. Myeloma patients with multiple day transplantation procedures had later neutrophil and platelet engraftment, longer hospitalization duration, more frequently febrile episodes, and inferior OS and RFS. ${ }^{10}$ Therefore, multiple day transplantation procedures assign myeloma patients to a high-risk group, deserving careful follow-up. Whereas one is tempted to recommend such patients prolonged maintenance treatment, ${ }^{15}$ one might anticipate poor tolerance due to the reduced HSC pool in these patients. However, such conclusions should be tested in a prospective manner. Alternatives to multiple day transplantation procedures, e.g. reduction of the DSMO dose in the autografts in patients with high transplant volume, merit to be evaluated.

\section{Acknowledgements}

The authors wish to thank the data management, the apheresis, the flow cytometry and the stem cell laboratory teams of the ASCT program at the University Hospital of Bern and its associated partner hospitals and collaborators for documentation of data relevant for this study.

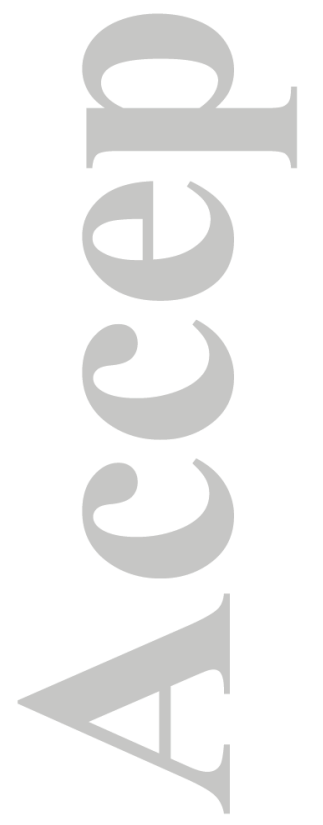




\section{References}

1. Palumbo A, Cavallo F, Gay F, et al. Autologous Transplantation and Maintenance Therapy in Multiple Myeloma. N Engl J Med. 2014;371(10):895-905.

2. Halle $\mathrm{P}$, Tournilhac $\mathrm{O}$, Knopinska-Posluszny $\mathrm{W}$, et al. Uncontrolled-rate freezing and storage at$80^{\circ} \mathrm{C}$, with only 3.5-percent DMSO in cryoprotective solution for 109 autologous peripheral blood progenitor cell transplantations. Transfusion, 2001;41(5):667-673.

3. Galmes A, Gutiérrez A, Sampol A, et al. Long-term hematological reconstitution and clinical evaluation of autologous peripheral blood stem cell transplantation after cryopreservation of cells with $5 \%$ and $10 \%$ dimethylsulfoxide at -80 degrees $\mathrm{C}$ in a mechanical freezer. Haematologica. 2007:92(7):986-989.

4. Morris C, de Wreede L, Scholten M, et al. Should the standard dimethyl sulfoxide concentration be reduced? Results of a European Group for Blood and Marrow Transplantation prospective noninterventional study on usage and side effects of dimethyl sulfoxide. Transfusion. 2014; 54(10):2514-2522.

5. Perseghin P, Balduzzi A, Bonanomi S, et al. Infusion-related side-effects in children undergoing autologous hematopoietic stem cell transplantation for acute leukemia. Bone Marrow Transplant. 2000;26(1):116-118.

6. Wuchter P, Ran D, Bruckner T, et al. Poor Mobilization of Hematopoietic Stem Cells-Definitions, Incidence, Risk Factors, and Impact on Outcome of Autologous Transplantation. Biol Blood Marrow Transplant. 2010;16(4):490-499.

7. Syme R, Bewick M, Stewart D, Porter K, Chadderton T, Glück S. The role of depletion of dimethyl sulfoxide before autografting: on hematologic recovery, side effects, and toxicity. Biol Blood Marrow Transplant. 2004;10(2):135-141.

8. Calmels B, Houzé P, Hengesse JC, Ducrot T, Malenfant C, Chabannon C. Preclinical evaluation of an automated closed fluid management device: Cytomate TM , for washing out DMSO from hematopoietic stem cell grafts after thawing. Bone Marrow Transplant. 2003;31(9):823-828.

9. Windrum $P$, Morris TC, Drake MB, Niederwieser D, Ruutu T. Variation in dimethyl sulfoxide use in stem cell transplantation: a survey of EBMT centres. Bone Marrow Transplant. 2005; 36(7):601-603.

10. Raschle J, Ratschiller D, Mans S, Mueller BU, Pabst T. High levels of circulating CD34+ cells at autologous stem cell collection are associated with favourable prognosis in multiple myeloma. Br J Cancer. 2011;105(7):970-974.

This article is protected by copyright. All rights reserved. 


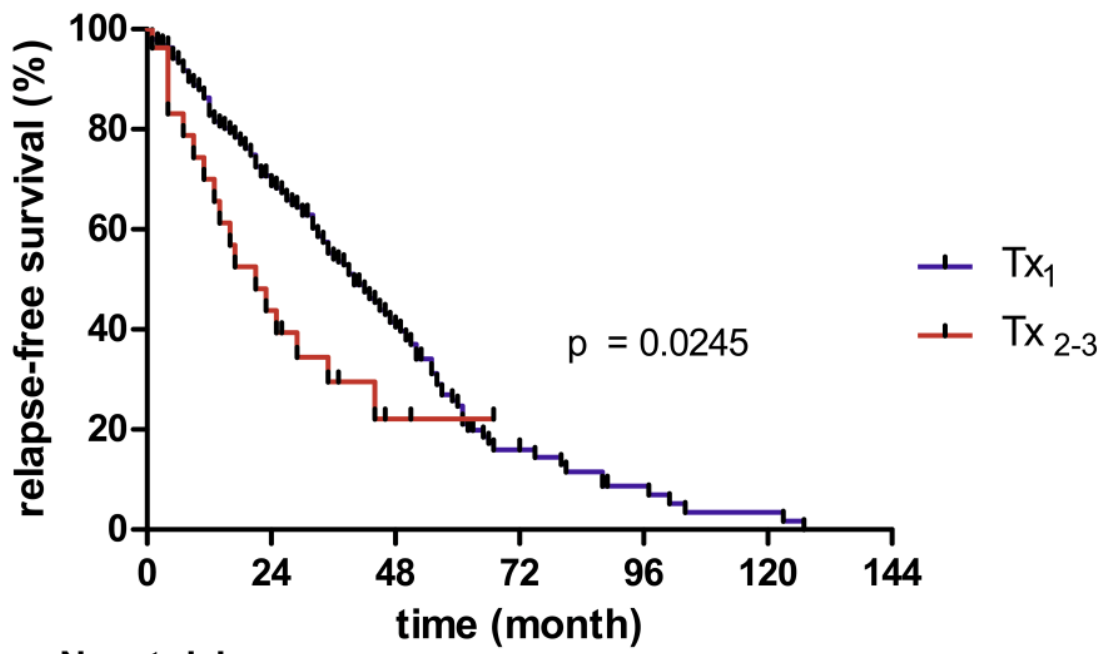

No. at risk

$\begin{array}{ccccccc}244 & 150 & 49 & 12 & 5 & 2 & T x_{1} \\ 27 & 11 & 2 & 0 & 0 & 0 & T x_{2-3}\end{array}$

Relapse-free survival after ASCT on one vs. multiple days

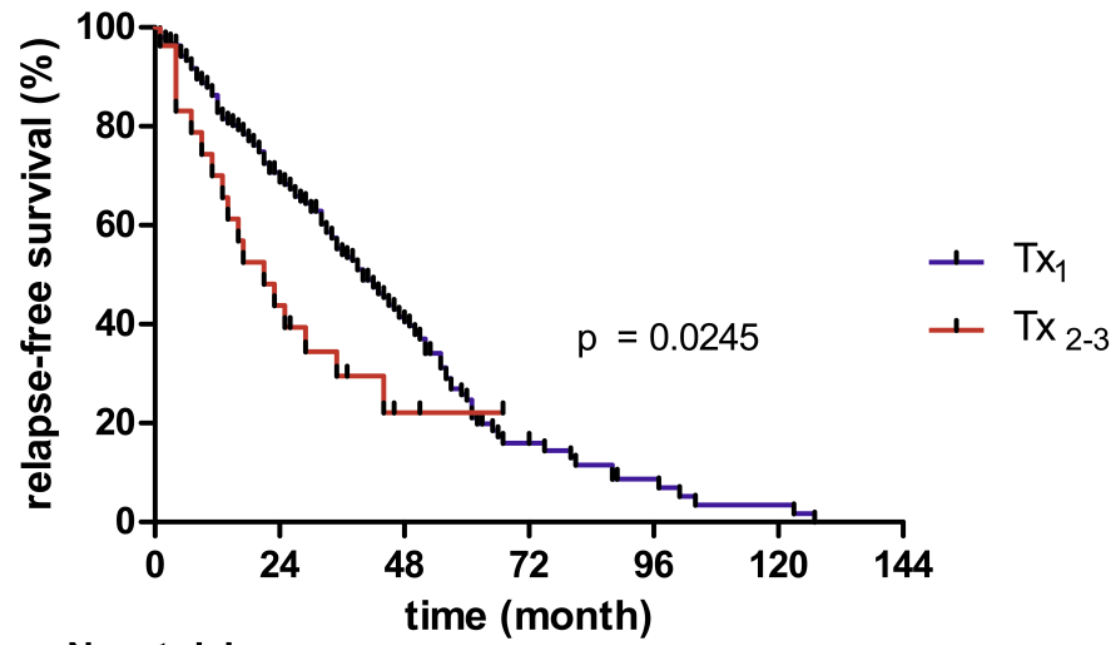

No. at risk

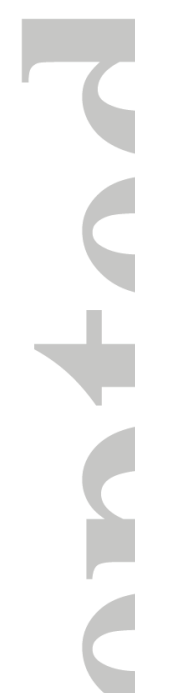

$\begin{array}{ccccccc}244 & 150 & 49 & 12 & 5 & 2 & T x_{1} \\ 27 & 11 & 2 & 0 & 0 & 0 & T x_{2-3}\end{array}$


Survival after ASCT on one vs. multiple days

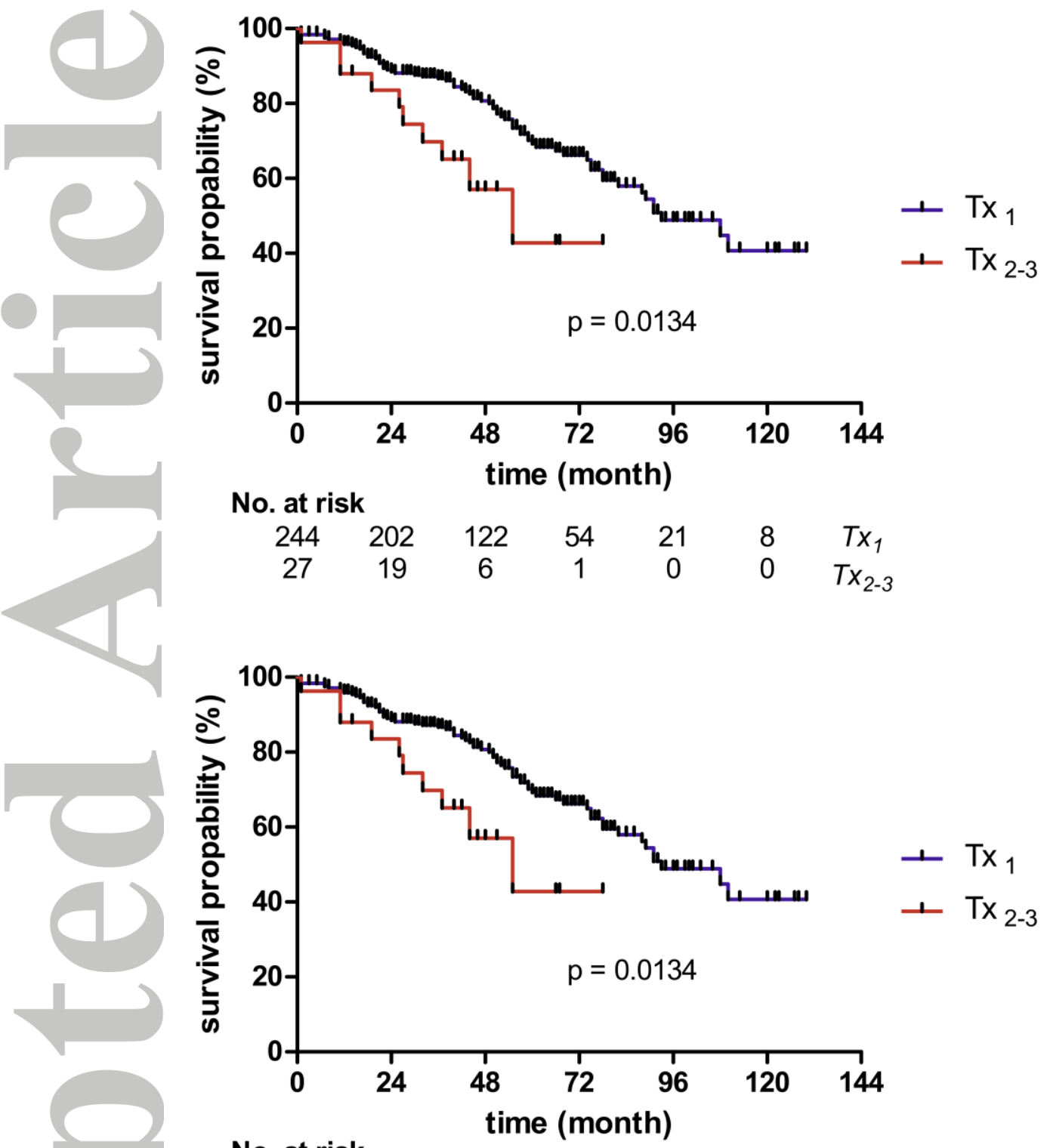

No. at risk

No. at risk

$\begin{array}{ccccccc}244 & 202 & 122 & 54 & 21 & 8 & T x_{1} \\ 27 & 19 & 6 & 1 & 0 & 0 & T x_{2-3}\end{array}$

Figure 1: Relapse-free (PFS) and overall survival (OS). (A) RFS and (B) OS of myeloma patients is compared between patients receiving the ASCT as a single-day procedure $\left(\mathrm{Tx}_{1}\right)$ as compared to patients receiving the autograft split over two or three days $\left(\mathrm{Tx}_{2-3}\right)$. 